\title{
Non-Markovian Infection Spread Dramatically Alters the Susceptible-Infected-Susceptible Epidemic Threshold in Networks
}

\author{
P. Van Mieghem* and R. van de Bovenkamp \\ Faculty of Electrical Engineering, Mathematics and Computer Science, Delft University of Technology, \\ P. O. Box 5031, 2600 GA Delft, The Netherlands \\ (Received 28 September 2012; published 5 March 2013)
}

\begin{abstract}
Most studies on susceptible-infected-susceptible epidemics in networks implicitly assume Markovian behavior: the time to infect a direct neighbor is exponentially distributed. Much effort so far has been devoted to characterize and precisely compute the epidemic threshold in susceptible-infected-susceptible Markovian epidemics on networks. Here, we report the rather dramatic effect of a nonexponential infection time (while still assuming an exponential curing time) on the epidemic threshold by considering Weibullean infection times with the same mean, but different power exponent $\alpha$. For three basic classes of graphs, the Erdős-Rényi random graph, scale-free graphs and lattices, the average steady-state fraction of infected nodes is simulated from which the epidemic threshold is deduced. For all graph classes, the epidemic threshold significantly increases with the power exponents $\alpha$. Hence, real epidemics that violate the exponential or Markovian assumption can behave seriously differently than anticipated based on Markov theory.
\end{abstract}

DOI: 10.1103/PhysRevLett.110.108701

PACS numbers: $89.75 . \mathrm{Hc}$

The epidemic threshold of a network distinguishes between the overall-healthy network regime and the effective infection regime where permanently a nonzero fraction of the nodes is infected. The epidemic threshold reflects the effectivity of an epidemic in a particular network and is a major indicator or tool to protect the nodes (people, computers, etc.) and to take preventive measures (governmental immunization strategies, antivirus software protection).

Recently (see, e.g., Refs. [1-7]) much effort has been devoted to the precise computation of the epidemic threshold in the continuous-time susceptible-infected-susceptible (SIS) Markov model in networks. In that simple SIS model, the viral state of a node $i$ at time $t$ is specified by a Bernoulli random variable $X_{i}(t) \in\{0,1\}: X_{i}(t)=0$ for a healthy node and $X_{i}(t)=1$ for an infected node. A node $i$ at time $t$ can be in one of two states: infected, with probability $v_{i}(t)=\operatorname{Pr}\left[X_{i}(t)=1\right]$ or healthy, with probability $1-v_{i}(t)$, but susceptible to the infection. The curing process per node $i$ is a Poisson process with rate $\delta$ and the infection rate per link is a Poisson process with rate $\beta$. Obviously, only when a node is infected, can it infect its direct neighbors that are still healthy. Both the curing and infection Poisson processes are independent. The network is represented by an adjacency matrix $A$, where $a_{i j}=1$ if there is a link from node $i$ to node $j$, otherwise $a_{i j}=0$. A major complication in the SIS Markov model is the absorbing state to which the epidemic SIS process always converges after a sufficiently long time in any network $G$ with a finite number $N$ of nodes and $L$ of links. Hence, the steady state is the overall-healthy (absorbing) state. Since the exact steady state is physically less meaningful, the epidemic threshold refers to the metastable or quasistationary state which is observed in practice. However, the metastable state needs to be defined (see, e.g., Refs. $[4,8]$ ).

Since $X_{i}$ is a Bernoulli random variable with the nice property that $E\left[X_{i}\right]=\operatorname{Pr}\left[X_{i}=1\right]$, the exact SIS governing equation [3] for node $i$ equals

$$
\begin{aligned}
\frac{d E\left[X_{i}(t)\right]}{d t}= & E\left[-\delta X_{i}(t)+\beta\left(1-X_{i}(t)\right) \sum_{k=1}^{N} a_{k i} X_{k}(t)\right] \\
= & E\left[-\delta X_{i}(t)+\beta \sum_{k=1}^{N} a_{k i} X_{k}(t)\right. \\
& \left.-\beta \sum_{k=1}^{N} a_{k i} X_{i}(t) X_{k}(t)\right],
\end{aligned}
$$

where (1) also holds for asymmetric adjacency matrices. In some of our previously published work, where undirected graphs (i.e., $A=A^{T}$ ) were assumed, $A$ must in some cases be replaced by its transpose $A^{T}$ in the equations in order to be valid for directed graphs. Directly from (1), we deduce that

$$
\frac{d E\left[X_{i}(t)\right]}{d t} \leq-\delta E\left[X_{i}(t)\right]+\beta \sum_{k=1}^{N} a_{k i} E\left[X_{k}(t)\right]
$$

When written for all nodes $i$ with $w_{i}=E\left[X_{i}(t)\right]$ and the vector $W=\left(w_{1}, w_{2}, \ldots, w_{N}\right)$, we obtain the matrix inequality

$$
\frac{d W(t)}{d t} \leq\left(\beta A^{T}-\delta I\right) W(t)
$$

from which

$$
W(t) \leq e^{\left(\beta A^{T}-\delta I\right) t} W(0)=e^{\left(\tau A^{T}-I\right) t^{*}} W(0),
$$


where the effective infection rate $\tau=\frac{\beta}{\delta}$ and the normalized time $t^{*}=\delta t$ is measured in units of the curing rate $\delta$. The upper bound is dominated by the fastest growth in $t^{*}$, which is due to the largest eigenvalue of $\tau A^{T}-I$. The exponential factor is dominated by $\tau \lambda_{1}(A)-1$, where $\lambda_{1}(A)$ is the real, largest eigenvalue of the non-negative matrix $A$ (by the Perron-Frobenius theorem; see Ref. [9]). When $\tau \lambda_{1}(A)-1 \leq 0$ or $\tau \leq \frac{1}{\lambda_{1}(A)}=\tau_{c}^{(1)}$, where $\tau_{c}^{(1)}$ is the first-order mean-field epidemic threshold [10], $w_{i}=$ $E\left[X_{i}(t)\right]$ decreases exponentially in $t^{*}$. Hence, the epidemic will die out fast. By definition of the epidemic threshold $\tau_{c}$ as the border between exponential die out and a nonzero fraction of infected nodes in the metastable state, we conclude that the exact epidemic threshold is $\tau_{c} \geq \tau_{c}^{(1)}$ in any finite sized network. The lower bound $\tau_{c}^{(1)}=\frac{1}{\lambda_{1}(A)}$ is of great practical use: if the effective infection rate $\tau$ can be controlled such that $\tau \leq \tau_{c}^{(1)}$, then the network is safeguarded from long-term, massive infection.

A basic property of a continuous-time Markov SIS process is the exponentially distributed infection time ([11], p. 184): a node $i$ infects its neighbors at an exponential time $T$ with mean $\frac{1}{\beta}$. Only the exponential distribution possesses the memoryless property which enables us to reduce the process history to only the previous event to compute the current one. Hence, all events in the past before the previous event are uncoupled as if the process restarts in the state at the previous event ([11], 349-351). Thus, the memoryless property of the exponential distribution makes Markovian processes attractive and analytically tractable. When a stochastic process is not Markovian, its mathematical description and analysis is considerably more complex. For example, the relatively simple and intuitive equation (1) and, more generally, the Chapman-Kolmogorov basic equations ([11], p. 180) that characterize Markov processes do not apply anymore. The (non-Markovian) time-dependent branching process [12], that is still tractable, exemplifies the increased mathematical complexity, while a non-Markovian epidemic model is analyzed in Ref. [13]. Hinrichsen [14] has surveyed the recent progress in the field of non-equilibrium phase transitions into absorbing states with long-range interactions and non-Markovian effects. In many non-Markovian processes on networks, computer simulation or measurement is often the only means to investigate its behavior and properties.

Here, we report the rather dramatic effect of a nonexponential infection time on the average steady-state fraction of infected nodes in networks. While the curing process is still Poissonian with rate $\delta$, the infection process at each node infects direct neighbors in a time $T$ that is Weibullean ([11], p. 56), with probability density function

$$
f_{T}(x)=\frac{\alpha}{b}\left(\frac{x}{b}\right)^{\alpha-1} e^{-(x / b)^{\alpha}}
$$

and mean $E[T]=b \Gamma\left(1+\frac{1}{\alpha}\right)$. In order to compare the Weibull with the exponential distribution, we fix the average infection time to $\frac{1}{\beta}$, so that

$$
b=\left(\Gamma\left(1+\frac{1}{\alpha}\right) \beta\right)^{-1} .
$$

Thus, the parameter $\alpha$ in (3) tunes the power-law start and the tail of the Weibull distributions that all have the same mean infection time $E[T]=\frac{1}{\beta}$, but variance equal to

$$
\operatorname{Var}[T]=\frac{1}{\beta^{2}}\left(\frac{\Gamma\left(1+\frac{2}{\alpha}\right)}{\Gamma^{2}\left(1+\frac{1}{\alpha}\right)}-1\right) .
$$

When $\alpha=1$, the Weibull distribution reduces to the exponential distribution. For $\alpha<1$, the tail decreases slower, but the probability of small infection times increases as a power law, proportional to $x^{\alpha-1}$. In the extreme limit for $\alpha \rightarrow 0$, the Weibull distribution tends to the Zipf distribution. In addition to the natural generalization of the exponential distribution, the Weibull distribution also recently appeared in epidemic studies [15].

The SIS process with Weibullean infection times is simulated as explained in Refs. [4,8]: the complicating absorbing state is removed so that the infection always remains in the network. When only one node is infected, the process restarts. At the precise moment that the last infected node is cured, that same node is reinfected. In order to determine the steady state of the fraction of infected nodes we run two simultaneous, but independent simulations on the same network. One simulation is initialized with a fraction of infected nodes equal to $10 \%$, whereas the second simulation starts with all nodes infected. During the simulation the time averaged number of infected nodes is measured. After running for 100000 state changes (or events) per simulation, we start comparing the average number of infected nodes in the two simulations and conclude that the steady state is reached when $\frac{\left|\bar{y}_{1}(t)-\bar{y}_{2}(t)\right|}{\bar{y}_{1}(t)+\bar{y}_{2}(t)}<10^{-4}$, where $\bar{y}_{1}(t)$ is the average fraction of infected nodes in the first simulation as a function of time and $\bar{y}_{2}(t)$ that of the second. The steady-state fraction of infected nodes is taken to be $\frac{\bar{y}_{1}(t)+\bar{y}_{2}(t)}{2}$.

Figure 1 shows the average steady-state fraction $y_{\infty}(\tau)=\lim _{t \rightarrow \infty} \frac{1}{N} E\left[\sum_{j=1}^{N} X_{j}(t)\right]$ of infected nodes in an Erdős-Rényi (ER) random graph $G_{p}(N)$ with $N=500$ nodes and with link density $p=2 p_{c}$, where the disconnectivity threshold ([11], p. 338) equals $p_{c} \sim \frac{\log N}{N}$, versus the effective infection rate $\tau$ for various $\alpha$, both larger and smaller than 1 . The curves $y_{\infty}(\tau)$ clearly shift to the right with increasing $\alpha$. More dramatically, when $\alpha$ decreases, the epidemic threshold also decreases. Not only does the threshold decrease, the smaller $\alpha$ the quicker the steadystate fraction of infected nodes increases when the epidemic threshold is passed. In other words, the derivative $\frac{d}{d \tau} y_{\infty}(\tau)$ increases with decreasing $\alpha$ for $\tau$ above the 


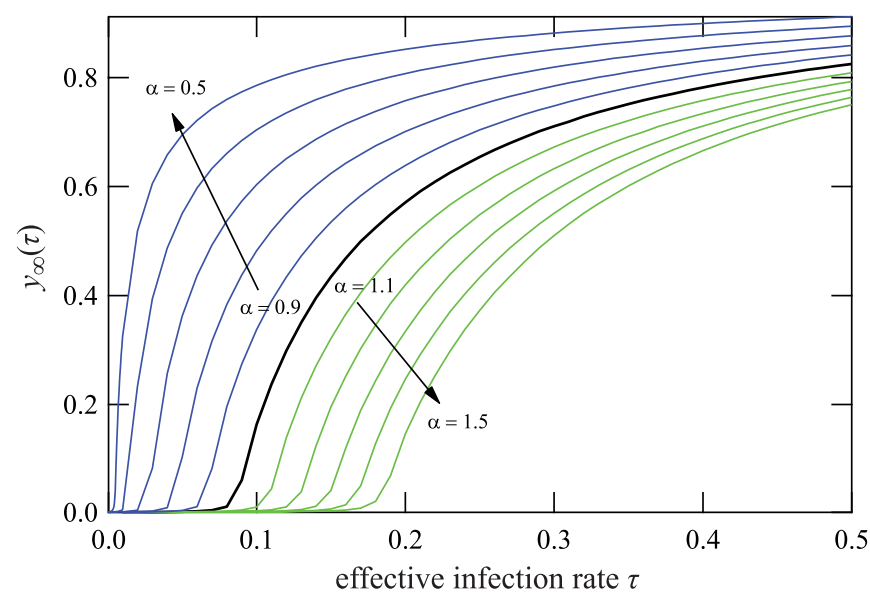

FIG. 1 (color online). Steady-state fraction of infected individuals as a function of the effective infection rate for various Weibull exponents on ER networks $\left(N=500, p=2 p_{c}\right.$, $d_{a}=12.4$ ).

epidemic threshold: the epidemic infects a larger number of nodes for a same average strength $\tau$. Hence, in nonMarkovian SIS epidemics with the same mean infection time (and same effective infection rate $\tau$ ), the sensitivity of the power law for values of $\alpha$ smaller than 1 on the epidemic threshold is large. A similar tendency is found in other types of graphs, for example, a scale-free (SF) graph (Fig. 2) and a rectangular grid (Fig. 3). As SF graph, we have simulated a Barabási-Albert graph, whose initial topology is a ring with $m$ nodes and where $m$ is the number of links added per newly added node and chosen in such a way that the resulting $\mathrm{SF}$ graph has approximately the same number of links as $G_{2 p_{c}}(N)$.

In the case of the grid (Fig. 3), the curves for successively smaller values of $\alpha$ are spaced further apart, while for larger values of $\alpha$ the opposite holds.

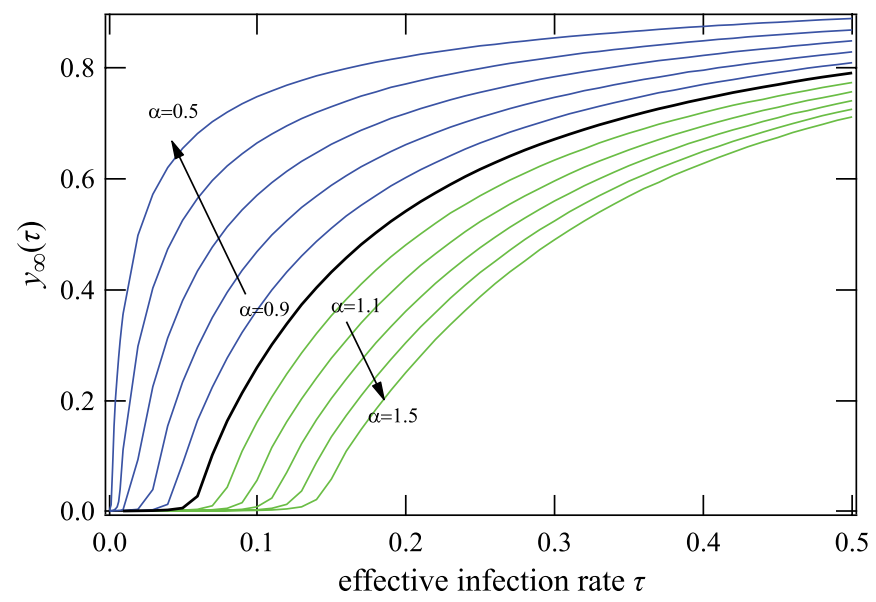

FIG. 2 (color online). Steady-state fraction of infected individuals as a function of the effective infection rate for various Weibull exponents on SF networks $(N=500, L=2970$, $d_{a}=11.8$ ).

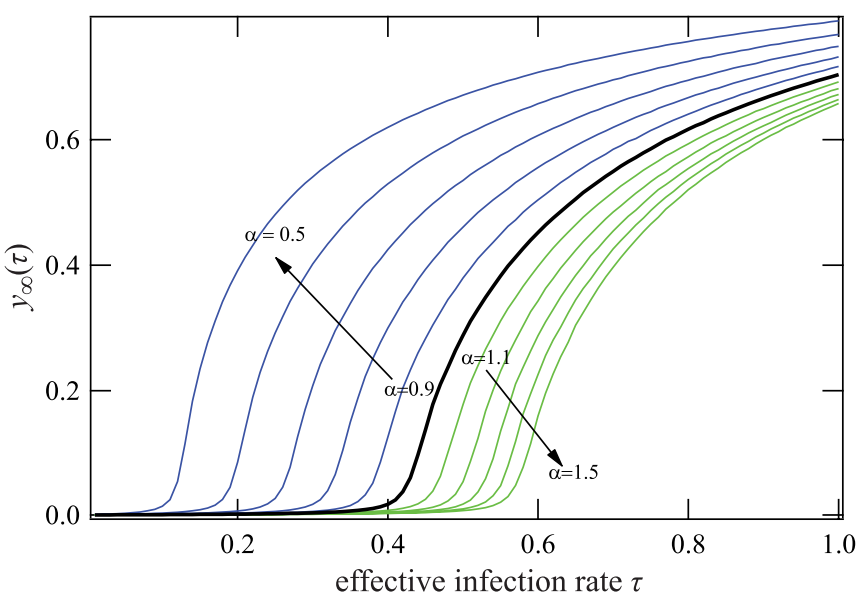

FIG. 3 (color online). Steady-state fraction of infected individuals as a function of the effective infection rate for various Weibull exponents on a rectangular grid $(N=484, L=924$, $d_{a}=3.8$ ).

For small values of $\alpha<1$, the Weibull pdf $f_{T}(t)$ in Fig. 4 shows that small infection times are more likely to occur, implying that many short infection attempts are fired to neighboring nodes, interchanged with a relatively long inactive time (because the mean $E[T]$ is constant). The exponential curing process is less effective to counteract the fast infection attempts leading to more infected nodes [higher $y_{\infty}(\tau)$ at a same $\tau$ ], but can clearly cope with the long inactive times, during which the neighbors cure and become healthy. Similarly, when $\alpha>1$, a quick succession of curing events takes place more frequently than infection attempts, thereby shifting $y_{\infty}(\tau)$ to larger effective infection rates $\tau$. Figure 4 draws the measured probability density function of the infection time $T$ for various values of the exponent $\alpha$. The infection time is only logged if it leads to a spreading action, that is, if it is smaller than the current curing time of the infected node. In all simulations the curing time is exponentially distributed with a mean of 1 (i.e., $\delta=1$ and $\tau=\beta$ ). As the infection time cannot be

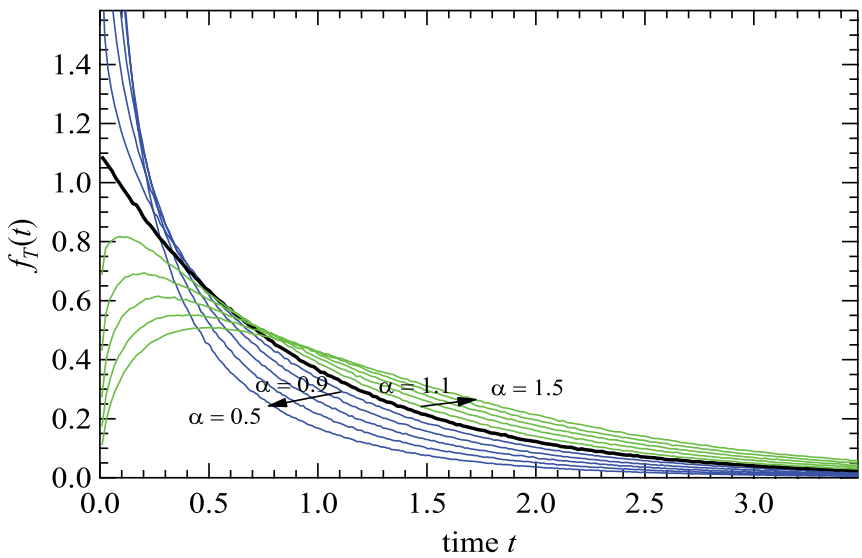

FIG. 4 (color online). The effectively simulated probability density function $f_{T}(t)$ of the infection $T$ for various $\alpha$. 


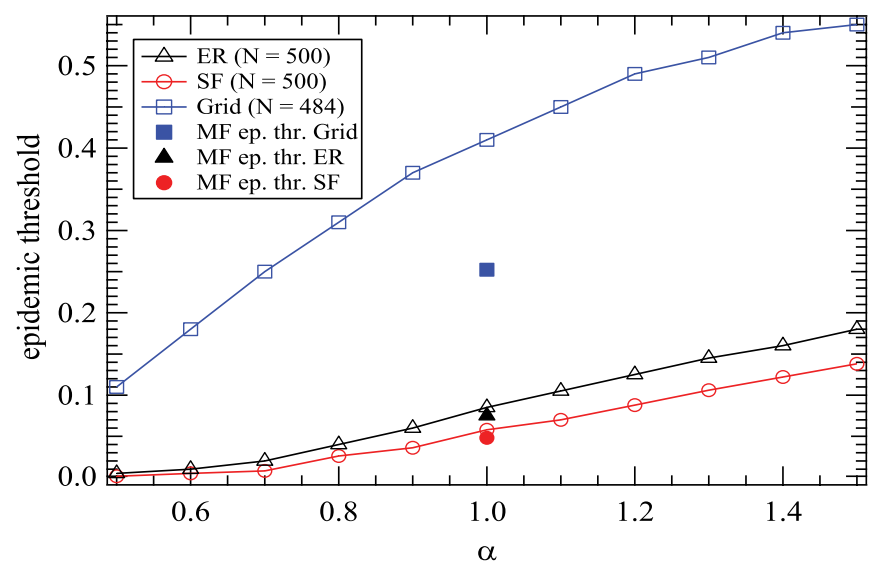

FIG. 5 (color online). The epidemic threshold, deduced from Figs. 1-3, versus the parameter $\alpha$ for the three types of graphs with approximately the same number of nodes $N$. The full markers correspond to the first-order mean-field epidemic threshold $\tau_{c}^{(1)}=\frac{1}{\lambda_{1}(A)}$ for $\alpha=1$.

larger than the cure time of the infected node, the infection time distribution is cut off for larger values by the exponential distribution of the cure time. Since the area below the curves equals one, by definition of a probability density function, each curve for $\alpha \neq 1$ intersects with the exponential distribution $(\alpha=1)$ twice. If $\alpha<1$, then small as well as very long infection times are more probable than for the exponential distribution, while the opposite holds for $\alpha>1$ as can be seen from Fig. 4. The thick line in Fig. 4 depicts the exponential case of $\alpha=1$. For each value of $\alpha$ approximately $7.5 \times 10^{6}$ infection times are logged.

Figure 5 illustrates how the epidemic threshold varies as a function of $\alpha$. For the ER and SF graph, the epidemic threshold is approximately linear for $\alpha>1$ and convex for $\alpha<1$, while the grid exhibits an opposite trend (linear for $\alpha<1$ and concave for $\alpha>1$ ). In addition to the larger effect of $\alpha$ on the epidemic threshold in lattices, the SIS epidemics in grids or lattices behaves, indeed, different from that of the denser graphs (in which the average degree increases with $N$ ). Also, mean-field approximations are less accurate for lattices than for the other two classes [8]. Finally, Fig. 6 shows that the epidemic threshold $\tau_{c}$ for ER and SF graphs as a function of the inverse spectral radius $\lambda_{1}$ (for various sizes of $N$ ranging from $N=10^{3}$ up to $64 \times 10^{3}$ ) are all power laws, suggesting that

$$
\tau_{c}(\alpha)=\frac{q(\alpha)}{\lambda_{1}^{r(\alpha)}}
$$

For the simulated $\alpha \in[0.5,1.5]$ and for both ER and SF graphs, we found that the exponent in (4) is approximately $r(\alpha) \approx \frac{1}{\alpha}$, but that $q(\alpha)$ is less accurate and not monotonic in $\alpha$. For $\alpha=1, q(1) \simeq 1.2$ indicating that the $\mathrm{N}$-intertwined mean-field approximation (NIMFA, [10])

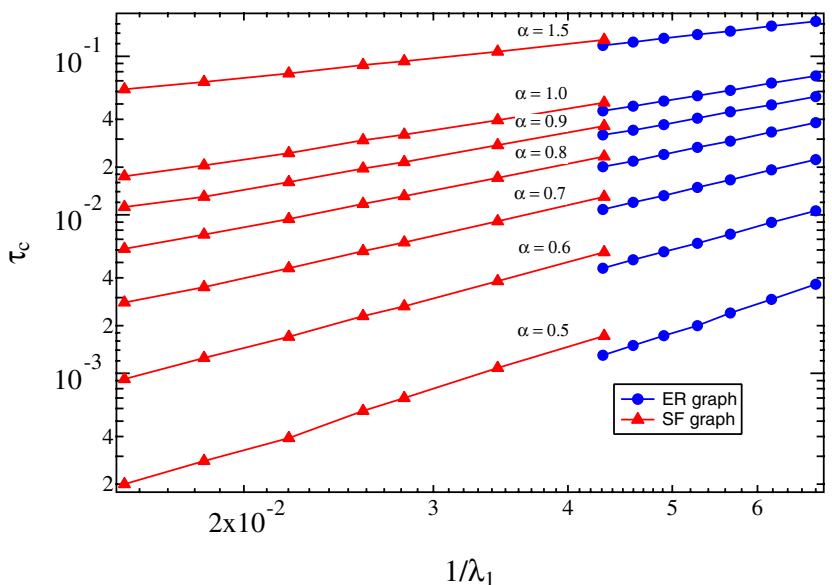

FIG. 6 (color online). The epidemic threshold in ER and SF graphs for various sizes $\left(N=2^{k} 10^{3}, 0 \leq k \leq 6\right)$ and value of $\alpha=(0.5,1.0,1.0)$ versus the inverse of the spectral radius of the graph.

lower bound $\tau_{c}^{(1)}=\frac{1}{\lambda_{1}}$ is about $20 \%$ smaller (for the considered graphs). The corresponding scaling of the grid with $N$ is different: all considered sizes (above $N=1000$ ) have the same epidemic threshold $\tau_{c}(\alpha)$ (because $\lambda_{1}$ is about 4 for all $N \geq 1000$ and nearly independent of $N$ ), but $\tau_{c}(\alpha)$ depends on $\alpha: \tau_{c}(0.5) \simeq 0.11, \tau_{c}(1) \simeq 0.41$ and $\tau_{c}(1.5) \simeq 0.56$. Based on the general bound [9], $\max \left(d_{\mathrm{av}}, \sqrt{d_{\max }}\right) \leq \lambda_{1} \leq d_{\max }$, where $d_{\mathrm{av}}$ and $d_{\max }$ are the average and maximum degree in a graph, respectively, Chung et al. [16] have proved for SF graphs with degree distribution $\operatorname{Pr}[D \geq x] \sim x^{-\gamma}$ that $\lambda_{1} \sim \sqrt{d_{\max }}=$ $O\left(N^{(1 / 2 \gamma)}\right)$ and Krivelevich and Sudakov [17] that $\lambda_{1}=O(\ln N)$ for $G_{p_{c}}(N)$. Combining these exact scaling laws with the law for $\tau_{c}(\alpha)$ in (4) suggests to us to conclude that the epidemic threshold in non-Markovian SIS epidemics will vanish with $N$ as a power law in SF graphs and as $O\left(\ln ^{-(1 / \alpha)} N\right)$ in ER networks.

In conclusion, the significant effect of a nonexponential infection time on the average steady-state fraction $y_{\infty}(\tau)$ of infected nodes and, thus on the epidemic threshold, questions the huge efforts to precisely compute the epidemic threshold in Markovean SIS epidemics in networks if viruses in real epidemics (or in computer networks) do not infect in an exponential time. Unfortunately, it appears exceedingly difficult to measure accurately the infection time in real epidemics to verify the exponential assumption made in almost all earlier SIS computations. From interactions with epidemiologists, rare measurements [18], and email activity [19], it seems quite likely that the infection time is not exponentially distributed, so that our observations here may point to a complete revision of SIS epidemics on networks. If real epidemics are not infecting direct neighbors in an exponential time, the study of the dependence of the epidemic threshold with the specifics of the measured infected time for various network classes and 
sizes stands on the agenda for future research. Our figures present initial results, but a more in-depth analysis is needed that covers more types of graphs and other heavy-tailed distributions for both infection and curing.

*P.F.A.VanMieghem@tudelft.nl

[1] C. Castellano and R. Pastor-Satorras, Phys. Rev. Lett. 105, 218701 (2010).

[2] C. Castellano and R. Pastor-Satorras, Sci. Rep. 2, 371 (2012).

[3] E. Cator and P. Van Mieghem, Phys. Rev. E 85, 056111 (2012).

[4] E. Cator and P. Van Mieghem, Phys. Rev. E 87, 012811 (2013).

[5] O. Givan, N. Schwartz, A. Cygelberg, and L. Stone, J. Theor. Biol. 288, 21 (2011).

[6] P. L. Simon, M. Taylor, and I.Z. Kiss, Math. Med. Biol. 62, 479 (2011).

[7] P. Van Mieghem and E. Cator, Phys. Rev. E 86, 016116 (2012).
[8] C. Li, R. van de Bovenkamp, and P. Van Mieghem, Phys. Rev. E 86, 026116 (2012).

[9] P. Van Mieghem, Graph Spectra for Complex Networks (Cambridge University Press, Cambridge, England, 2011).

[10] P. Van Mieghem, J. Omic, and R.E. Kooij, IEEE/ACM Trans. Netw. 17, 1 (2009).

[11] P. Van Mieghem. Performance Analysis of Communications Systems and Networks (Cambridge University Press, Cambridge, England, 2006).

[12] T.E. Harris, The Theory of Branching Processes (Springer-Verlag, Berlin, 1963).

[13] A. N. Startsev, Theory Probab. Appl. 41, 730 (1997).

[14] H. Hinrichsen, J. Stat. Mech. (2007) P07006.

[15] G. Streftaris and G. J. Gibson, Biostatistics 13, 580 (2012).

[16] F. Chung, L. Lu, and V. Vu, Proc. Natl. Acad. Sci. U.S.A. 100, 6313 (2003).

[17] M. Krivelevich and B. Sudakov, Comb. Probab. Comput. 12, 61 (2003).

[18] G. L. Yang, Math. Biosci. 14, 65 (1972).

[19] A. Vazquez, B. Rácz, A. Lukács, and A.-L. Barabási, Phys. Rev. Lett. 98, 158702 (2007). 